\title{
CALIDAD FISIOLÓGICA, ÁCIDOS GRASOS Y RESPIRACIÓN EN SEMILLAS DE TOMATE DE CÁSCARA DETERIORADAS ARTIFICIALMENTE
}

\author{
PHYSIOLOGICAL QUALITY, FATTY ACIDS CONTENT AND RESPIRATION OF HUSK \\ TOMATO SEEDS ARTIFICIALLY DETERIORATED
}

\author{
Juan M. Pichardo González ${ }^{1}$, Óscar J. Ayala Garay ${ }^{*}$, Víctor A. González Hernández ${ }^{1}$, César M. Flores \\ Ortiz $^{2}$, J. Alfredo Carrillo Salazar ${ }^{1}$, Aureliano Peña Lomeli ${ }^{3}$, Alejandrina Robledo Paz ${ }^{1}$ y Gabino García de \\ los Santos ${ }^{1}$
}

\begin{abstract}
${ }^{1}$ Postgrado en Recursos Genéticos y Productividad, Colegio de Postgraduados, Campus Montecillo. Km. 36.5 Carretera México-Texcoco. 56230, Montecillo, Texcoco, Estado de México. Tel. 01 (595) 952-0200. Ext. 1594. ${ }^{2}$ Laboratorio de Biogeoquímica, FES-Iztacala, Universidad Nacional Autónoma de México. Av. de los Barrios No. 1, Los Reyes Iztacala. 54090, Tlalnepantla, Estado de México. ${ }^{3}$ Departamento de Fitotecnia, Universidad Autónoma Chapingo. Km. 38.5 Carretera México-Texcoco. 56230, Chapingo, Texcoco, Estado de México.
\end{abstract}

* Autor para correspondencia (oayala@colpos.mx)

\section{RESUMEN}

Durante el almacenamiento, mecanismos de deterioro de la semilla disminuyen la germinación, la velocidad de crecimiento de la plántula y la tolerancia a condiciones adversas. El deterioro se puede evaluar con pruebas de envejecimiento artificial (EA). En este trabajo se evaluó el deterioro artificial de semilla de tomate de cáscara (Physalis ixocarpa Brot.) de la variedad 'CHF1-Chapingo', y la respuesta germinativa se correlacionó con el contenido de ácidos grasos totales y la tasa de respiración de semillas completas. En un primer experimento se utilizaron semillas envejecidas artificialmente en 55, 65 y $75 \%$ de humedad relativa (HR) a 45 y $50{ }^{\circ} \mathrm{C}$, durante 30 d; se evaluó la germinación (G, \%), el vigor (velocidad de emergencia de radícula, VER, radículas $\mathrm{d}^{-1}$ ), y el contenido de ácidos grasos cuantificado mediante cromatografía con espectrómetro de masas. En un segundo experimento se utilizaron semillas envejecidas artificialmente en 50, 55, 60, 65, 70, 75 y $80 \%$ de $\mathrm{HR}$ a $50{ }^{\circ} \mathrm{C}$, durante 30 d y se evaluó germinación, VER, y actividad respiratoria a 72 h de imbibición. Se encontró que el ácido linoleico fue el de mayor concentración (87-93 \% del total de ácidos grasos) en estas semillas, y que el aumento de calor y humedad redujo $(\mathrm{P} \leq 0.5)$ la $\mathrm{G}$ y la VER, pero no al contenido de ácidos grasos. En el Experimento 1 la combinación de $50{ }^{\circ} \mathrm{C}$ y $65 \%$ de HR disminuyó la $\mathrm{G}$ en $53 \%$ y a la VER en $92 \%$, con respecto al testigo no tratado. En el experimento 2, el tratamiento de $50{ }^{\circ} \mathrm{C}$ con $65 \%$ de HR disminuyó la $\mathrm{G}$ en $21 \%$ t la VER en $78 \%$, con respecto al tratamiento de $50{ }^{\circ} \mathrm{C}$ con $50 \%$ de HR. La actividad respiratoria correlacionó con la disminución de germinación $(r=0.88)$ y de VER $(r=0.99)$, por lo cual la pérdida de la calidad fisiológica de la semilla se atribuye a la disminución en la tasa de respiración.

Palabras clave: Physalis ixocarpa, ácidos grasos, deterioro artificial de semillas, respiración.

\section{SUMMARY}

During storage, seeds deteriorate due to mechanisms that reduce their germination rate, seedling growth rate and tolerance to adverse conditions. Seed deterioration can be measured by means of artificial aging tests (AA). In this research the artificial deterioration of husk tomato seeds (Physalis ixocarpa Brot.) var. 'CHF1Chapingo' was evaluated, and the germination rate was correlated with fatty acids content and respiratory activity. In a first experiment, seeds were artificially aged by applying 55,65 and $75 \%$ of relative humidity $(\mathrm{RH})$ at 45 and $50{ }^{\circ} \mathrm{C}$, for $30 \mathrm{~d}$ and germination rate $(G, \%)$, seed vigor (radical emergence rate, RER) and fatty acids content (quantified by means of a gas chromatograph connected to a mass spectrometer) were evaluated. In a second experiment, seeds were aged by imposing $50,55,60,65,70,75$ and $80 \%$ of $\mathrm{RH}$ at $50{ }^{\circ} \mathrm{C}$ for $30 \mathrm{~d}$; G, RER and respiration rate (measured after $72 \mathrm{~h}$ of imbibition) were measured as response variables. It was found that linoleic acid was the most abundant fatty acid (87-93\% of the total content) in these seeds, and the increase of heat and relative humidity caused a significant reduction $(P \leq 0.05)$ in $G$ and SER without affecting fatty acids content. Compared to untreated seeds (control), the combined effect of $50{ }^{\circ} \mathrm{C}$ and $65 \%$ RH produced losses of $53 \%$ in G and of $92 \%$ in RER, in Experiment 1. The same treatment $\left(50{ }^{\circ} \mathrm{C}\right.$ and $\left.65 \% \mathrm{RH}\right)$ induced losses of $21 \%$ in $\mathbf{G}$ and of $78 \%$ in RER when compared to responses at $50{ }^{\circ} \mathrm{C}$ and $50 \% \mathrm{RH}$. The respiratory rate correlated with the losses in $G(r=0.88)$ and RER $(r=0.99)$, thus indicating that loss of physiological quality in deteriorated seeds can be attributed to the reduction in respiratory activity.

Index words: Physalis ixocarpa, fatty acids, artificial seed deterioration, respiration.

\section{INTRODUCCIÓN}

El deterioro de semillas está relacionado con su edad, contenido de humedad, y condiciones de almacenamiento; es un proceso en el cual disminuyen la germinación, la velocidad de crecimiento de plántulas y la tolerancia a condiciones adversas. Los síntomas de la semilla deteriorada incluyen: crecimiento anormal, daños en las 
estructuras principales de las plántulas, pérdida de compuestos solubles (debido a excesiva permeabilidad de la membrana), reducción de la actividad enzimática, daño oxidativo al ADN y proteínas, y producción de sustancias tóxicas (Bradford, 2004).

Los enlaces dobles de las cadenas de los ácidos grasos son vulnerables a las reacciones con agentes oxidantes fuertes, como peróxido de hidrógeno $\left(\mathrm{H}_{2} \mathrm{O}_{2}\right)$, radical aniónico superóxido $\left(\mathrm{O}_{2}\right)$ y radical hidroxilo $(\mathrm{OH})$, que son formas tóxicas que oxidan los ácidos grasos y los convierten en hidroperóxidos (ROOH). Los lípidos de las membranas celulares se oxidan más fácilmente pues contienen abundantes ácidos grasos insaturados. La estructura y funcionamiento de la membrana se modifica cuando el daño oxidativo se extiende a las proteínas de la membrana (Melo y Cuamatzi, 2008), lo que genera cambios en su permeabilidad, pérdida de fluidez e integridad (Dell'Aquilla, 1994).

La falta de integridad de las membranas celulares de las semillas se atribuye a la disminución de fosfolípidos, carbohidratos y proteínas, así como a la menor actividad de la enzima peroxidasa durante el secado natural que ocurre en la maduración (Wallis et al., 2000). La peroxidación lipídica daña las semillas, en tres formas: destrucción de lípidos membranales, oxidación de radicales libres y formación de aldehídos citotóxicos que pueden inactivar proteínas (Bradford, 2004). Como consecuencia, los ácidos grasos de las membranas disminuyen conforme la semilla se deteriora y pierde capacidad germinativa y vigor (Wilson y McDonald, 1986; Walters et al., 2005; Panobianco y Viera, 2007).

El mayor deterioro de la semilla también se asocia con la disminución de carbohidratos que generalmente ocurre al envejecer la semilla, por que los sustratos respiratorios para la germinación resultan insuficientes o existe incapacidad para usarlos. Por tanto un signo del deterioro de la semilla es la disminución significativa de su tasa respiratoria (Cruz-Pérez et al., 2003).

Las pruebas de laboratorio de deterioro controlado o envejecimiento acelerado se usan para evaluar la calidad fisiológica de las semillas, con el supuesto de que el proceso de deterioro artificial es similar al deterioro natural (Nogarajan y Pandita, 2001). Estas pruebas (ISTA, 2004) se aplican principalmente en especies agrícolas de semilla grande (granos). En semillas pequeñas, como en flores, hortalizas y pastos (Halmer, 2000) se ha encontrado una correlación baja entre el envejecimiento artificial y la calidad fisiológica; sin embargo, no se han estudiado suficientes variantes de temperatura y humedad de deterioro en estas semillas.
México es centro de origen de especies del género Physallis, y en 2006 el tomate de cáscara (Physalis ixocarpa Brot.) ocupó el quinto lugar en producción entre las especies hortícolas, con un millón de toneladas (SIAP, 2006). A pesar de su importancia, es una especie todavía poco estudiada y la investigación relacionada con los aspectos de deterioro y calidad de semilla es escasa. Pérez-Camacho et al. (2008) estimaron una tasa anual de pérdida de germinación de $8.7 \%$ por envejecimiento natural, la cual es superior a la de $7.6 \%$ encontrada en tomate [Lycopersicon lycopersicum (L.) Karsten ex Farw.] (Doijode, 1987), e inferior a la de $13.2 \%$ reportada en chile (Capsicum annuum L.) (Thakur et al., 1988). Las razones de la pérdida de germinación en tomate de cáscara no han sido aún clarificadas.

El objetivo de este trabajo fue determinar el efecto del deterioro artificial de semilla de $P$. ixocarpa en germinación, vigor, composición de ácidos grasos y respiración.

\section{MATERIALES Y MÉTODOS}

\section{Experimento 1}

En Chapingo, Estado de México, en invernadero con cubierta de plástico, se sembró la variedad 'CHF1Chapingo' el 6 de noviembre de 2006. La cosecha de frutos fue en la última semana de enero de 2007. La semilla extraída del fruto se secó a temperatura ambiente bajo sombra y se benefició (limpieza y selección por tamaño) en una máquina de aire-zarandas (tipo LALS, marca Kamas ${ }^{\circledR}$, Industri AB, Suecia). La semilla beneficiada se almacenó en bolsas de papel, en una bodega sin control de clima $\left(18.2 \pm 5{ }^{\circ} \mathrm{C}\right.$ de temperatura y $41 \pm 10 \%$ de HR).

Durante 2007 se evaluaron seis tratamientos de envejecimiento acelerado aplicados a la semilla, más un testigo (sin deterioro inducido). Los seis tratamientos de envejecimiento resultaron de tres grados de $\operatorname{HR}(55,65 \mathrm{y}$ $75 \%)$ combinados con dos temperaturas $\left(45\right.$ y $\left.50{ }^{\circ} \mathrm{C}\right)$. Para inducir los niveles deseados de HR, se utilizaron recipientes de plástico de $267 \mathrm{~mL}$, en cuyo interior se colocaron cajas Petri que contenían $5 \mathrm{~g}$ de semilla. Enseguida se agregaron soluciones saturadas de cloruro de litio $(\mathrm{LiCl})$ que se prepararon con $15.8,12.6$ y $9.6 \mathrm{~g}$ de $\mathrm{LiCl}$ y luego aforados a $50 \mathrm{~mL}$. Las soluciones se colocaron dentro del recipiente de forma que rodearan a la caja Petri con la semilla. Los recipientes se taparon herméticamente y se pusieron a 45 y $50{ }^{\circ} \mathrm{C}$ en estufa durante $30 \mathrm{~d}$ (Walters y Ross, 1993). El testigo fue semilla proveniente de la citada bodega, cuyo contenido 
de humedad fue $6 \%$, calculado con la fórmula de Bewley y Black (1994).

Al término de los tratamientos de deterioro artificial se evaluó el porcentaje de germinación $(\mathrm{G})$ y la velocidad de emergencia de radícula (VER, radículas $\mathrm{d}^{-1}$ ), en tres repeticiones de 50 semillas por tratamiento (ambiente), con un diseño completamente al azar con siete tratamientos y tres repeticiones. La siembra se hizo en agar bacteriológico estéril a $1 \%$, en una germinadora SD8900® (Seedburo Inc., USA) programada con $12 \mathrm{~h}$ luz y $12 \mathrm{~h}$ oscuridad (radiación $80 \mu \mathrm{mol} \mathrm{s} \mathrm{m}^{-1}$ ), $70 \%$ de HR y $28 \pm 2{ }^{\circ} \mathrm{C}$ de temperatura, por $9 \mathrm{~d}$. La VER (expresión del vigor de la semilla) se calculó con la fórmula de Maguire (1962), que se basa en el número de radículas emergidas cada $24 \mathrm{~h}$.

Igualmente, en un diseño completamente al azar con tres repeticiones se evaluó la composición de los siguientes ácidos grasos: heptadecanoico, linoleico, oleico y palmítico. La extracción y esterificación fue mediante el método de Priestley y Leopold (1979), y la reacción de estratificación por el método de Morrison y Smith modificado por Priestley et al. (1985). Para determinar la composición de ácidos grasos se utilizó un cromatógrafo de gases acoplado a un espectrómetro de masas, ambos marca Finnigan MAT CAQ ${ }^{\circledR}$ (Alemania). Las condiciones cromatográficas fueron: inyección, modo de "dispersión de partícula"; temperatura del inyector, 200 ${ }^{\circ} \mathrm{C}$; temperaturas del horno, programada desde $100{ }^{\circ} \mathrm{C}(3$ min al inicio) hasta $250{ }^{\circ} \mathrm{C}$ (por $4 \mathrm{~min}$ ) con aumentos de $5{ }^{\circ} \mathrm{C} \min ^{-1}$; columna del cromatógrafo, tipo DB-1 (Dimetilpolisiloxano); helio como gas acarreador, con una velocidad de $35 \mathrm{~cm} \mathrm{~s}^{-1}$. Las condiciones del detector de masas fueron: temperatura de la línea de transferencia, $250{ }^{\circ} \mathrm{C}$; intervalo de 20 a 400 reacciones de masa-carga $(\mathrm{m} / \mathrm{z})$; polaridad positiva; temperatura de la fuente de iones, $200{ }^{\circ} \mathrm{C}$; ionización de impacto electrónico, $70 \mathrm{eV}$. Se inyectaron $2 \mu \mathrm{L}$ de la fase orgánica del extracto. Los estándares metílicos de los ácidos grasos se identificaron con los tiempos de retención de estándares y con los patrones de fragmentación de bibliotecas de referencia NIST (National Institute of Standars and Technology).

\section{Experimento 2}

El objetivo de este experimento fue precisar los niveles de HR que combinados con una temperatura de $50{ }^{\circ} \mathrm{C}$ causan deterioro artificial de la semilla de tomate de cáscara. Se utilizó semilla de la misma variedad, producida en campo en Chapingo, México, cuya siembra fue en la primera semana de marzo de 2007 y la cosecha en la última semana de junio del mismo año. La extracción, secado, beneficio y almacenamiento de las semillas fueron iguales que en el Experimento 1. La semilla sometida al envejecimiento acelerado en septiembre de 2007 , tenía $7 \%$ de humedad.

La prueba de envejecimiento acelerado se hizo a una sola temperatura $\left(50^{\circ} \mathrm{C}\right)$, con siete grados de $\operatorname{HR}(50,55$, $60,65,70,75$ y $80 \%$ ), que se generaron con $17.5,15.8$, $14.2,12.6,11.1,9.6$ y $8.1 \mathrm{~g}$ de $\mathrm{LiCl}$ aforados a $50 \mathrm{~mL}$ de agua destilada. La cantidad de semilla, tipo de recipientes, repeticiones, diseño experimental y condiciones de temperatura, luz y humedad de la germinadora, fueron las mismas que en el Experimento 1. Como testigo se utilizó semilla no envejecida artificialmente. La prueba de germinación se hizo en papel filtro.

Además del porcentaje de germinación (G) y la velocidad de emergencia de radícula, se midió la cinética respiratoria de la semilla con un cromatógrafo de gases modelo HP 5890® (Hewlet Packard, EE. UU.). La respiración se midió a las $72 \mathrm{~h}$ de iniciado el proceso de imbibición-germinación, en tres repeticiones de 50 semillas en diseño completamente al azar. Las 50 semillas se colocaron en frascos de vidrio de $36 \mathrm{~mL}$ que después se cerraron herméticamente con un tapón de plástico. El $\mathrm{CO}_{2}$ de cada frasco se midió después de $1 \mathrm{~h}$ de tapado; se extrajo $1 \mathrm{~mL}$ de aire con una jeringa para insulina, el cual se inyectó en el cromatógrafo. $\mathrm{El} \mathrm{CO}_{2}$ liberado por respiración se calculó con el área bajo la curva de cada muestra. Los datos se transformaron a $\mathrm{mL} \mathrm{h}^{-1}$.

En ambos experimentos los análisis estadísticos se hicieron con el programa SAS (SAS Institute, 2002). Las variables medidas en porcentaje se transformaron con la fórmula arco seno $\sqrt{X / 100}$. Las comparaciones de medias fueron mediante Tukey (0.05). Se aplicó análisis de regresión del porcentaje de germinación y velocidad de emergencia de la radícula sobre la cinética de respiración. Las curvas sigmoidales se ajustaron a los datos observados con el programa Curve Expert 1.3. Las gráficas se elaboraron en la hoja de cálculo Microsoft Excel 2007® (Microsoft Corp., USA).

\section{RESULTADOS Y DISCUSIÓN}

\section{Experimento 1}

Los análisis de varianza mostraron que los tratamientos de envejecimiento afectaron $(\mathrm{P} \leq 0.01)$ la germinación y la velocidad de emergencia de radícula (VER), pero no la composición de ácidos grasos de las semillas de tomate de cáscara, excepto al ácido palmítico $(\mathrm{P} \leq 0.05)$ (Cuadro 1). Los coeficientes de variación de G y VER fueron bajos ( $\leq$ $15 \%)$, mientras que los de los ácidos grasos fueron altos 
(28 a $77 \%$ ), lo que se atribuye a la heterogeneidad de las semillas en estas variables.

La semilla del testigo (sin envejecimiento artificial) tuvo mayor germinación y velocidad de emergencia de la radícula que la de los tratamientos de envejecimiento acelerado (Cuadro 2). La G disminuyó en promedio $48 \%$ y la VER en $85 \%$ al subir la temperatura de $45{ }^{\circ} \mathrm{C}$ a 50 ${ }^{\circ} \mathrm{C}$. En las condiciones más estresantes (temperatura de $50{ }^{\circ} \mathrm{C}$ y humedad relativa de $75 \%$ ) la capacidad germinativa y vigor fueron mínimos $(7.3 \%$ para $\mathrm{G}$ y 0.4 radículas $\mathrm{d}^{-1}$ para VER). El incremento en la $\mathrm{HR}$ a temperatura constante de $45{ }^{\circ} \mathrm{C}$ no redujo significativamente la $\mathrm{G}$, y ésta se mantuvo por arriba del $85 \%$ establecido en norma (ISTA, 2004). En cambio, el aumento de $\mathrm{HR}$ en $50{ }^{\circ} \mathrm{C}$ provocó una disminución constante en la germinación, hasta bajar a $7 \%$ con $75 \%$ de HR, lo que evidenció un efecto significativo de la combinación de ambos factores en el deterioro de las semillas. Bhering et al. (2006), en semilla de Capsicum frutescens L. sometida a envejecimiento acelerado, encontraron que la aplicación de $45^{\circ} \mathrm{C}$ en periodos de 72 a $96 \mathrm{~h}$ a $100 \%$ de HR provocó efectos más drásticos que un periodo prolongado de exposición al envejecimiento sin calor.
Según Salinas et al. (2001), los resultados de la prueba de germinación pueden utilizarse para clasificar el vigor de los lotes de semilla. Así, los lotes de semilla envejecidos artificialmente que presenten una germinación superior a $80 \%$ podrían considerarse como de alto vigor, entre 60 y $80 \%$ como de vigor medio, y menores de 60 $\%$ como de bajo vigor. Con base en esta clasificación, se puede inferir que el testigo, las semillas envejecidas a 45 ${ }^{\circ} \mathrm{C}$ y las tratadas a $50{ }^{\circ} \mathrm{C}$ con $55 \%$ de HR mantienen un alto nivel de germinabilidad ( $>80 \%)$ y por tanto tendrían alto vigor, mientras que las semillas tratadas a 50 ${ }^{\circ} \mathrm{C}$ y $\mathrm{HR} \geq 65 \%$ pierden vigor $(<60 \%$ en germinabilidad).

El mayor índice de velocidad de emergencia también correspondió al testigo, con 25.7 radículas $\mathrm{d}^{-1}$ (Cuadro 2). $\mathrm{Al}$ igual que en la variable $\mathrm{G}$, el incremento de la $\mathrm{HR}$ a temperatura constante de $45{ }^{\circ} \mathrm{C}$ no redujo tan notoriamente la VER como el incremento en la HR a temperatura de $50{ }^{\circ} \mathrm{C}$, donde con $55 \%$ de HR la VER disminuyó a 6.5 radículas $\mathrm{d}^{-1}$ (pérdida de $75 \%$ con respecto al testigo) y a 0.4 radículas $\mathrm{d}^{-1}$ (pérdida de $98 \%$ con respecto al testigo) con $75 \%$ de HR.

Cuadro 1. Cuadrados medios y significancia estadística en germinación, velocidad de emergencia de radícula y contenido de ácidos grasos de semillas de tomate de cáscara, en siete tratamientos de deterioro artificial.

\begin{tabular}{|c|c|c|c|c|c|c|c|}
\hline$\overline{\mathrm{FV}}$ & $\mathrm{gl}$ & $\begin{array}{c}\text { VER } \\
\text { (radículas d }^{-1} \text { ) }\end{array}$ & $\begin{array}{c}\mathrm{G} \\
(\%)\end{array}$ & \multicolumn{4}{|c|}{$\begin{array}{l}\text { Contenido de ácidos grasos } \\
\left(\mathrm{mg} \mathrm{g}^{-1} \text { de semilla) }\right.\end{array}$} \\
\hline Tratamientos & 6 & $309.9 * *$ & $1964.8 * *$ & $1.0 *$ & 4039.9 & 22.4 & 0.1 \\
\hline CV (\%) & & 14.0 & 10.0 & 28.0 & 66.0 & 77.0 & 38.0 \\
\hline
\end{tabular}

$*, * *$ significativo con $\mathrm{P} \leq 0.05$ y $\mathrm{P} \leq 0.01$ respectivamente; $\mathrm{FV}=$ fuentes de variación; $\mathrm{CV}=$ coeficiente de variación; gl $=$ grados de libertad; $\mathrm{G}=$ germinación; VER = velocidad de emergencia de radícula.

Cuadro 2. Efecto de calor y humedad relativa en germinación, vigor y contenido de ácidos grasos, en de semillas de tomate de cáscara sometidas a tratamientos de deterioro artificial (Exp. 1).

\begin{tabular}{|c|c|c|c|c|c|c|c|}
\hline \multirow[t]{2}{*}{$\begin{array}{l}\text { Temp. } \\
{ }^{\circ} \mathrm{C}\end{array}$} & \multirow[t]{2}{*}{$\begin{array}{l}\mathrm{HR} \\
(\%)\end{array}$} & \multirow[t]{2}{*}{$\begin{array}{l}\mathrm{G} \\
(\%)\end{array}$} & \multirow[t]{2}{*}{$\begin{array}{c}\text { VER } \\
\left.\text { (radículas d }^{-1}\right)\end{array}$} & \multicolumn{4}{|c|}{$\begin{array}{c}\text { Contenido de ácidos grasos } \\
\left(\mathrm{mg} \mathrm{g}^{-1} \mathrm{de} \text { semilla }\right)\end{array}$} \\
\hline & & & & Palmítico & Linoleico & Oleico & Esteárico \\
\hline 18 & (Testigo) & 98.7 a & $25.7 \mathrm{a}$ & $1.8 \mathrm{ab}$ & $46.0 \mathrm{a}$ & $3.3 \mathrm{a}$ & $1.0 \mathrm{a}$ \\
\hline 45 & 55 & $95.0 \mathrm{a}$ & $19.6 \mathrm{~b}$ & $1.5 \mathrm{~b}$ & $56.8 \mathrm{a}$ & $2.7 \mathrm{a}$ & $0.7 \mathrm{a}$ \\
\hline 45 & 65 & $94.7 \mathrm{a}$ & $21.0 \mathrm{ab}$ & $1.2 \mathrm{~b}$ & $35.1 \mathrm{a}$ & $2.7 \mathrm{a}$ & $0.4 \mathrm{a}$ \\
\hline 45 & 75 & $88.3 \mathrm{a}$ & $18.7 \mathrm{~b}$ & $1.6 \mathrm{ab}$ & $32.9 \mathrm{a}$ & $2.5 \mathrm{a}$ & $0.7 \mathrm{a}$ \\
\hline 50 & 55 & $82.0 \mathrm{a}$ & $6.5 \mathrm{c}$ & $1.2 \mathrm{~b}$ & $78.7 \mathrm{a}$ & $4.1 \mathrm{a}$ & $0.5 \mathrm{a}$ \\
\hline 50 & 65 & $46.0 \mathrm{~b}$ & $2.1 \mathrm{~cd}$ & $1.3 \mathrm{~b}$ & $77.3 \mathrm{a}$ & $3.9 \mathrm{a}$ & $0.8 \mathrm{a}$ \\
\hline 50 & 75 & $7.3 \mathrm{c}$ & $0.4 \mathrm{~d}$ & $2.8 \mathrm{a}$ & $138.5 \mathrm{a}$ & $10.2 \mathrm{a}$ & $0.8 \mathrm{a}$ \\
\hline DMS & & 19.3 & 5.6 & 1.3 & 125.4 & 9.2 & 0.8 \\
\hline Promedio & & 73.1 & 13.4 & 1.7 & 66.5 & 4.2 & 0.7 \\
\hline
\end{tabular}

Medias con letras iguales no son estadísticamente diferentes (Tukey, 0.05); Temp. = temperatura; HR = humedad relativa; $\mathrm{G}=$ germinación;

VER $=$ velocidad de emergencia de radícula; DMS = diferencia mínima significativa. 
En general, estos resultados mostraron que el aumento de calor a $50{ }^{\circ} \mathrm{C}$ asociado con $\mathrm{HR} \geq 65 \%$, causa deterioro en el desempeño germinativo de las semillas de la variedad 'CHF1-Chapingo' de tomate de cáscara. Además, la disminución de los valores de G y VER (vigor) no estuvieron asociados con el contenido de ácidos grasos.

En cuanto a los ácidos grasos, el linoleico fue el de mayor concentración (de 87 a $93 \%$ del total), seguido del oleico (de 4.4 a $6.9 \%$ ), el palmítico (de 1.4 a $4.2 \%$ ) y del esteárico (de 0.6 a $2 \%$ ) (Cuadro 2, Figura 1); los dos primeros ácidos grasos son insaturados y los otros dos saturados. En semillas, los ácidos grasos insaturados se ubican primordialmente en la membrana celular (Walters et al., 2005; Gómez-Tejero et al., 2006; Panobianco y Vieira, 2007), por lo que se infiere que la mayor parte de los ácidos observados en las semillas analizadas, provienen de este tipo de membranas. Al respecto, PérezCamacho et al. (2008) encontraron que el principal compuesto de reserva de la semilla de tomate de cáscara var. 'CHF1-Chapingo' es el almidón y no observaron cuerpos lipídicos.

Los tratamientos incluidos en el grupo estadístico superior de ácido palmítico fueron el de $50{ }^{\circ} \mathrm{C}$ con $75 \%$ de HR (Tratamiento 6), el testigo no sometido a envejecimiento artificial y el Tratamiento $3\left(45^{\circ} \mathrm{C}\right.$ con $75 \%$ de HR). Puesto que en los Tratamientos 6 y 3 se aplicó la máxima HR, se puede inferir que una alta HR durante el almacenamiento afectará poco la concentración de este ácido graso en la semilla de esta variedad, y que las diferencias ocurrirían más bien en HR intermedias, de 55 a $65 \%$.

La hipótesis de la reparación metabólica establece que puede haber condiciones que contrarrestan los efectos nocivos del envejecimiento (Wilson y McDonald, 1986). Una hidratación previa o posterior puede prevenir o reparar los daños causados por los radicales libres, como consecuencia de la capacidad del agua para desactivarlos (McDonald, 1999). Como en este trabajo esto no se evaluó, queda para futuros estudios por verificar si a 75 $\%$ de HR hay una hidratación de la semilla que contrarreste el efecto dañino de los radicales libres y que este efecto sea más grande a $50{ }^{\circ} \mathrm{C}$ que a $45{ }^{\circ} \mathrm{C}$. Los ácidos linoleico, oleico y esteárico no mostraron cambios significativos por efecto de los tratamientos de envejecimiento acelerado (Cuadro 2).

En general, se esperaba una menor concentración de los ácidos grasos en la semilla más deteriorada, como propusieron Panobianco y Vieira (2007), y como Wilson y McDonald (1986) observaron en semilla de Lycopersicon esculentum Mill. Sin embargo, no siempre ocurre esto (McDonald, 1999), pues en semillas de Glicine max L. cvs. 'Chippewa 64' (Priestley y Leopold, 1979) y 'Century' más tres isolineas (Trawatha et al., 1995) y Helianthus annuus cv 'Briosol' (Bailly et al., 2002), no se detectó decremento en los ácidos grasos conforme se acentuó el deterioro de las semillas, posiblemente porque hubo reparación metabólica.

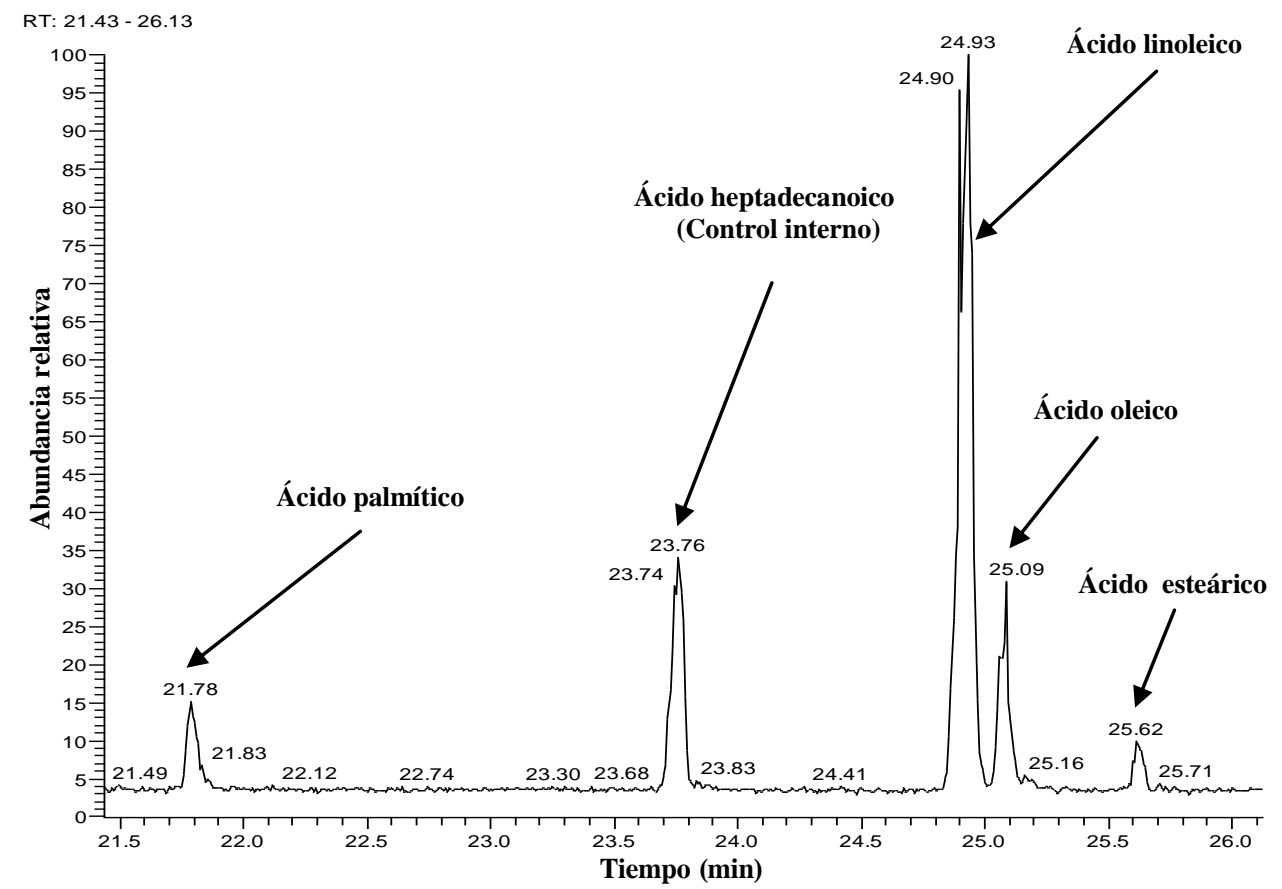

Figura 1. Cromatograma de ácidos grasos presentes en semillas de tomate de cáscara. $R T$ = tiempo de retención. 


\section{Experimento 2}

Con base en los resultados del Experimento 1, en el segundo experimento el deterioro artificial se indujo con siete niveles de HR todos aplicados a $50{ }^{\circ} \mathrm{C}$ a semillas de tomate de cáscara recién cosechadas en junio de 2007. El análisis de varianza (Cuadro 3) mostró que las humedades relativas con las que se provocó el envejecimiento acelerado afectaron $(\mathrm{P} \leq 0.01)$ a $\mathrm{G}$, a VER y a la actividad respiratoria medida a las $72 \mathrm{~h}$ de imbibición. Los coeficientes de variación en todas las variables fueron bajos $(<15 \%)$.

$\mathrm{Al}$ mantener constante la temperatura $\left(50{ }^{\circ} \mathrm{C}\right)$ y variar la HR desde 50 hasta $60 \%$ no hubo deterioro en la $\mathrm{G}$ (Figura 2A), la cual se mantuvo por arriba de $85 \%$, y estadísticamente similar al testigo $(\mathrm{P} \leq 0.05)$ cuya germinación fue $95 \%$. Cuando la HR fue igual o superior a $65 \%$ la germinación se redujo a $73 \%$. Resultados similares se observaron en el Experimento 1 (Cuadro 2). Conforme aumentó la HR, la $\mathrm{G}$ disminuyó aún más, de modo que con $70 \%$ de HR la G sólo fue $2 \%$; a partir de $75 \%$ de HR el deterioro artificial fue letal $(0 \%$ de G) para las semillas de esta variedad de tomate de cáscara.

Las mayores VER se registraron en el testigo $\left(18{ }^{\circ} \mathrm{C}\right.$ y $41 \% \mathrm{HR}$ ) y a $50{ }^{\circ} \mathrm{C}$ con $50 \%$ de HR (Figura $2 \mathrm{~B}$ ), con 18.7 radículas $\mathrm{d}^{-1}$; aún a $55 \%$ de HR se obtuvo un índice VER alto ( $>16$ radículas d), pero a $60 \%$ de HR la VER disminuyó significativamente, y fue nula a partir de $75 \%$ de HR. Es decir, G y VER disminuyeron conforme aumentó la humedad relativa con una cinética sigmoidal inversa, lo que demuestra que el incremento de este factor ambiental provoca deterioro de la semilla a $50{ }^{\circ} \mathrm{C}$. Tanto en este experimento como en el primero, la VER mostró el mismo comportamiento que la $\mathrm{G}$.

La mayor actividad respiratoria a $72 \mathrm{~h}$ de iniciada la imbibición fue con $50 \% \mathrm{HR}\left(0.67 \mathrm{~mL}\right.$ de $\left.\mathrm{CO}_{2} \mathrm{~g}^{-1} \mathrm{~h}^{-1}\right)$ (Figura 2C). La semilla seca del testigo, antes de embeberse, mostró una actividad respiratoria alta $(0.60$ $\mathrm{mL}$ de $\mathrm{CO}_{2} \mathrm{~g}^{-1} \mathrm{~h}^{-1}$ ) pero significativamente más baja que el punto mayor. La actividad respiratoria empezó a disminuir a $60 \%$ de HR y más rápidamente a $65 \%$ de $\mathrm{HR}$, hasta alcanzar una tasa muy baja $\left(0.5 \mathrm{~mL}\right.$ de $\mathrm{CO}_{2} \mathrm{~g}^{-1}$ $\mathrm{h}^{-1}$ ) a una HR de $75 \%$.
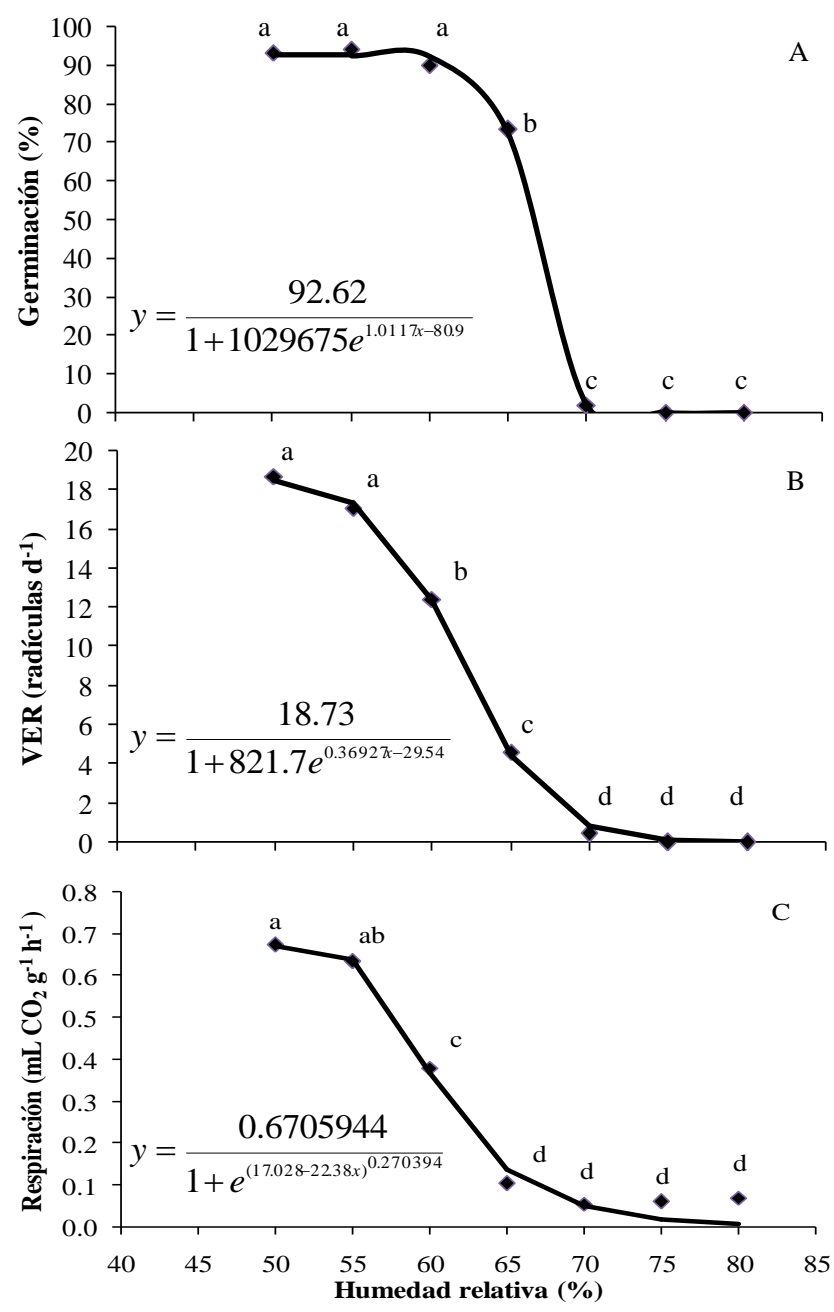

Figura 2. Efecto de la humedad relativa en el envejecimiento acelerado de semillas de tomate de cáscara almacenadas a $50{ }^{\circ} \mathrm{C}$ (Exp. 2). A) Germinación. ( $\left(\mathrm{n}=7 ; \mathrm{P}<0.01 ; \mathrm{R}^{2}=0.99\right)$. B) Velocidad de emergencia de la radícula (VER). $\left(n=7 ; P<0.01 ; R^{2}\right.$ $=0.99$ ), C) Cinética respiratoria de las semillas a las $72 \mathrm{~h}$ después de la imbibición. $\left(n=7 ; P<0.01 ; R^{2}=0.99\right.$. Valores con la misma letra son estadísticamente similares (Tukey, 0.05).

Cuadro 3. Cuadrados medios y significancia estadística de germinación, velocidad de emergencia de radícula y actividad respiratoria de semillas de tomate de cáscara envejecidas en siete niveles de $\mathrm{HR}$ a $50{ }^{\circ} \mathrm{C}$ (Exp. 2).

\begin{tabular}{lcccc}
\hline Fuente de variación & gl & $\begin{array}{c}\text { Germinación } \\
(\%)\end{array}$ & $\begin{array}{c}\text { VER } \\
(\text { radículas d }\end{array}$ & $\begin{array}{c}\text { Respiración } \\
\left(\mathrm{mL} \mathrm{de} \mathrm{CO}_{2} \mathrm{~g}^{-1} \mathrm{~h}^{-1}\right)\end{array}$ \\
\hline Tratamientos de EA & 7 & $3982.9 * *$ & $222.9 * *$ & $0.24 * *$ \\
Error & 14 & 12.7 & 1.6 & 0.0004 \\
$\mathrm{CV}(\%)$ & & 8.0 & 14.0 & 7.0 \\
\hline$* *$ Significativo con P $\leq$ 0.01; gl = grados & de libertad; & VER = velocidad de emergencia de radícula; $\mathrm{CV}=$ coeficiente de \\
variación.
\end{tabular}
variación. 
Lo anterior demuestra que la alta humedad relativa combinada con calor de $50{ }^{\circ} \mathrm{C}$ provocan decrementos de la respiración con la consecuente baja en la generación de energía metabólica requerida para la germinación, como De Visser et al. (1990) encontraron en haba (Vicia faba L.). En semilla de tomate de cáscara, Pérez-Camacho et al. (2008) encontraron que la disminución de reservas de carbohidratos explica en gran parte las pérdidas de germinación y vigor de la semilla.
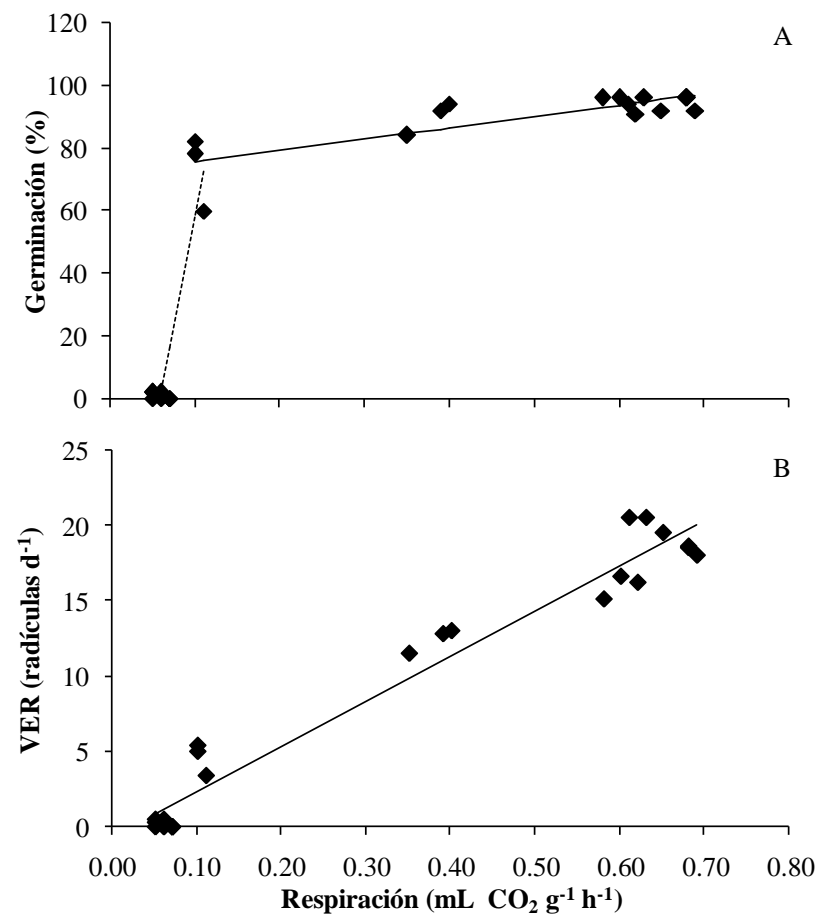

Figura 3. Cinética respiratoria de semillas a $72 \mathrm{~h}$ después del inicio de la imbibición (Exp. 2), sobre: A) Porcentaje de germinación. Si $x$ $\leq 0.11$, entonces $y=1410.5 x-82.252\left(n=12 ; P<0.01 ; R^{2}=\right.$ 0.77); si $x>0.11$, entonces $y=36.068 x+71.978(n=15 ; P<$ 0.01; $\left.\mathbf{R}^{2}=0.66\right)$. B) Velocidad de emergencia de radícula. $\mathbf{y}=$ 30.14x-0.72 $\left(n=24 ; P<0.01 ; R^{2}=0.95\right)$. En todos los casos " $x$ " es la tasa respiratoria de las semillas a $72 \mathrm{~h}$ después de iniciada la imbibición.

La actividad respiratoria a las $72 \mathrm{~h}$ (Figura 3) podría considerarse una prueba temprana de la calidad fisiológica de un lote de semillas, ya que se asocia linealmente con la germinación y con la velocidad de emergencia de la radícula. En la germinación se observa un punto crítico a $65 \%$ de HR cuando la tasa de respiración es $0.11 \mathrm{~mL}$ $\mathrm{CO}_{2} \mathrm{~g}^{-1} \mathrm{~h}^{-1}$; con tasas de respiración inferiores a este punto crítico se acelera la reducción de la germinación que baja de $76 \%$ a $0 \%$, mientras que con tasas superiores la germinación sólo aumenta de 76 a $95 \%$. Sundstrom y Edwars (1989) también propusieron que la actividad respiratoria en semillas de Capsicum annuum L. puede servir como un indicador confiable del vigor de semilla, ya que tasas altas de respiración correlacionaron con germinación alta y con una rapida emergencia de radícula.

Con base en los resultados se infiere que la disminución de la actividad respiratoria fue la principal causante de deterioro en la semilla de tomate de cáscara.

\section{CONCLUSIONES}

En semillas de tomate de cáscara var. 'CHF1Chapingo', el tratamiento de envejecimiento artificial de $50{ }^{\circ} \mathrm{C}$ combinado con $65 \%$ de humedad relativa provocó una disminución de $53 \%$ en la germinación y de $92 \%$ en la velocidad de emergencia de la radícula, con respecto al testigo no tratado. La velocidad de emergencia de radícula fue más sensible que la germinación, al incrementar la temperatura de 45 a $50 \quad{ }^{\circ} \mathrm{C}$. Los tratamientos de envejecimiento acelerado no cambiaron significativamente la composición de ácidos grasos (heptadecanoico, linoleico, oleico y palmítico) en las semillas. El acido linoleico fue el de mayor concentración en la semilla, al constituir entre 87 y $93 \%$ del total de ácidos grasos (32.9-138.5 mg g $\left.{ }^{-1}\right)$. La respiración de la semilla declinó a valores inferiores a $0.1 \mathrm{~mL}$ de $\mathrm{CO}_{2} \mathrm{~g}^{-1}$ $\mathrm{h}^{-1}$ a partir del tratamiento de $50{ }^{\circ} \mathrm{C}$ combinado con $65 \%$ de humedad relativa, lo que correlacionó positivamente con la pérdida de germinación $(\mathrm{r}=0.88)$ y con la velocidad de emergencia de radícula $(\mathrm{r}=0.99)$. El decremento de la calidad fisiológica se puede explicar por la disminución de la respiración. En esta variedad de tomate de cáscara el deterioro germinativo de las semillas no se asoció con cambios en el contenido de ácidos grasos de las membranas celulares.

\section{BIBLIOGRAFÍA}

Bailly C, R Bogatek-Leszczynska, D Come, F Corbineu (2002) Changes in activities of antioxidant enzymes and lipoxygenase during growth of sunflower seedlings from seeds of different vigour. Seed Sci. Res. 12:47-55.

Bewley J D, M Black (1994) Seeds. Physiology of Development and Germination. 2nd. ed. Plenum Press. New York, USA. 445 p.

Bhering M C, D C Fernandes D S D, D De Souza V, D D S Portocarrero N (2006) Teste de envelhecimiento acelerado em sementes de pimenta. Rev. Bras. Sementes 28:64-71.

Bradford K J (2004) Seed Production and Quality. Department of Vegetable Crop and Weed Science. University of California. Davis CA, USA. $134 \mathrm{p}$.

Cruz-Pérez A B, V A González-Hernández, M C Mendoza-Castillo, M L Ortega-Delgado (2003) Marcadores fisiológicos de la tolerancia al envejecimiento de semilla en maíz. Agrociencia $37: 371-381$.

Dell'Aquilla A (1994) Wheat seed ageing and embryo protein degradation. Seed Sci. Res. 4:293-298.

De Visser R, H M Dekhuijzen, D R Verkerke (1990) Control of seed respiration and growth in Vicia faba by oxygen and temperature: No evidence for an oxygen diffusion barrier. Plant Physiol. 93:668-672. 
Doijode S D (1987) Seed longevity in different tomato cultivars. Prog. Horti. 19:87-89

Gómez-Tejero J, J Jasso-Mata, J J Vargas-Hernández, M R SotoHernández (2006) Deterioro de semilla de dos procedencias de Swietenia macrophylla King., bajo distintos métodos de almacenamiento. Ra Ximhai 1:223-239.

Halmer $\mathbf{P}$ (2000) Comercial seed treatment technology. In: Seed Technology and its Biological Basis. M Black, J D Bewley (eds). Sheffield Academic Press. UK. pp:257-286.

ISTA, International Seed Testing Association (2004) International Rules for Seed Testing. Rules 2004. ISTA Ed. Zurich, Switzerland. $243 \mathrm{p}$.

Maguire J (1962) Speed of germination-aid in selection and evaluation for seedling emergence and vigor. Crop Sci. 2:176-177.

McDonald M B (1999) Seed deterioration: physiology, repair and assessment. Seed Sci. Technol. 27:177-237.

Melo R V, O Cuamatzi T (2008) Bioquímica de los Procesos Metabólicos. 2a ed. Ed. Reverté. México, D. F. 406 p.

Nogarajan S, V K Pandita (2001) Improvement in germination characteristics in artificially aged seeds tomato by osmoconditioning. Seed Res. 29:136-140.

Panobianco M, R D Vieira (2007) Electrical conductivity and deterioration of soybean seeds exposed to different storage conditions. Rev. Bras. Sementes 29:97-105.

Pérez-Camacho I, O J Ayala-Garay, V A González-Hernández, J A Carrillo-Salazar, A Peña-Lomelí, G García-de los Santos (2008) Indicadores morfológicos y fisiológicos del deterioro de semillas de tomate de cáscara. Agrociencia 42:891-901.

Priestley D A, A C Leopold (1979) Absence of lipid oxidation during accelerated aging of soybean seeds. Plant Physiol. 63:726-729.

Priestley D A, B Werner, A C Leopold, M McBride (1985) Organical free radical levels in seeds and pollen: The effects of hydration and aging. Physiol. Plant. 64:88-94.
Salinas A R, A M Yoldjian, R M Cravioto, V Bisaro (2001) Pruebas de vigor y calidad fisiológica de semillas de soja. Pesq. Agropec. Bras. 36:371-379.

SAS Institute (2002) User's Guide of SAS (Statistical Analysis System). SAS Institute Inc. Cary, N. C. USA. 550 p.

SIAP, Sistema Integral de Información Agroalimentaria y Pecuaria (2006) Anuario del Sistema Integral de Información Agroalimentaria y Pecuaria. Secreataría de Agricultura, Ganadería Desarrollo Rural, Pesca y Alimentación (SAGARPA). Disponible en : www.siap.sagarpa gob.mx/ar_comdeanuadim.html (Marzo 2007).

Sundstrom F J, R L Edwards (1989) Pepper seed respiration, germination, and seedling development following seed priming. HortScience 24:343-345.

Thakur P C, S Joshi, T S Verma, K S Kapoor (1988) Effect of storage period on germination of sweet pepper seed. Capsicum Newslet. 7:58-59.

Trawatha S E, D M TeKrony, D F Hildebrand (1995) Relationship of soybean seed quality to fatty acid and $\mathrm{C}_{6}$-aldehyde levels during storage. Crop Sci. 35:1415-1422.

Wallis J, J Shockey, J Browse (2000) Seed oils and their metabolic engineering. In: Seed Technology and its Biological Basis. M Black, J D Bewley (eds). Sheffield Academic Press. UK. pp:121-159.

Walters C, E Ross (1993) Theorical basis of protocols for seed storage II. The influence of temperature on optimal moisture levels. Seed Sci. Res. 3:201-213.

Walters C, P Landré, L Hill, F Corbineau, C Bailly (2005) Organization of lipid reserves in cotyledons of primed and aged sunflower seeds. Planta 222:397-407.

Wilson D O, M B McDonald (1986) The lipid peroxidation model of seed deterioration. Seed Sci. Technol. 14:269-300. 